J3eA, Journal sur l'enseignement des sciences et technologies de l'information et des systèmes, Volume 2, Hors-Série 2, 5 (2003)

DOI : http://dx.doi.org/10.1051/bib-j3ea:2003605

(C) EDP Sciences, 2003

Vers l'intégration de convertisseurs pour l'alimentation des microsystèmes

B. Estibals, J.-L. Sanchez, C. Alonso, H. Camon et J.-P. Laur

Laboratoire d'Analyse et d'Architecture des Systèmes (LAAS-CNRS)

7 avenue du Colonel Roche

F-31077 Toulouse Cedex 04, France

bestibalelaas.fr, sanchez@laas.fr, alonsocelaas.fr 


\title{
Vers l'intégration de convertisseurs pour l'alimentation des microsystèmes.
}

\author{
Bruno ESTIBALS, Jean-Louis SANCHEZ, Corinne ALONSO, Henri CAMON, Jean-Pierre LAUR. \\ Laboratoire d'Analyse et d'Architecture des Systèmes (LAAS-CNRS) \\ 7, avenue du Colonel Roche -31077 TOULOUSE Cedex 04
}

\author{
E-mail: . bestibal@laas.fr, sanchez@laas.fr, alonsocelaas.fr
}

\begin{abstract}
Résumé
Le travail présenté dans cet article a pour but de faire une synthèse des dernières avancées en matière de recherche sur l'intégration des convertisseurs d'énergie, servant d'étage d'adaptation entre des micro-sources d'énergie et des applications à faibles besoins énergétiques, comme les microsystèmes. Ce type d'applications présente des contraintes diverses en tension, en courant et fréquences. Après avoir établi une classification des problèmes à résoudre, nous proposons différentes pistes pour réaliser des fonctions de conversion.
\end{abstract}

\section{Introduction.}

La miniaturisation permanente des composants électroniques permet d'en embarquer de plus en plus dans les équipements et accessoires portables de grande consommation, tels que les téléphones cellulaires, les lecteurs MP3, les caméras, etc... Ces derniers deviennent multi-fonctionnels, pouvant par exemple cumuler la saisie et le traitement d'image, la reconnaissance vocale, les transmissions de données, ces fonctions faisant de plus en plus appel aux microsystèmes. Mais, ceci implique un nouveau besoin en sources d'énergie miniaturisées et performantes [1]. Si l'alimentation électrique n'a suscité que peu d'intérêts et d'efforts de recherche et de développement dans le passé, elle est aujourd'hui unanimement reconnue comme l'enjeu majeur à surmonter pour les prochaines générations de l'électronique portable [2].

Etant donnée l'activité de recherche au LAAS-CNRS sur les microsystèmes, et en particulier sur les MOEMS, nous nous sommes à notre tour intéressés aux divers types d'alimentation possibles pour ce type d'applications [3]. Pour cela, après avoir effectué une recherche sur les diverses sources d'énergie qui pourraient convenir aujourd'hui, nous nous sommes particulièrement intéressé aux traitements de ces énergies, et en particulier comment elles pourraient s'adapter aux applications par l'intermédiaire de convertisseurs statiques et de commandes adéquates. Ce domaine, faisant l'objet d'enjeux importants, est en perpétuelle évolution, tant en réduction des tailles des composants qu'en quantité d'énergie transférée. Cependant, un certain nombre de verrous technologiques existent encore pour en faire des produits fiables et commercialisables.
Comme, dès le départ, nous avons souhaité adopter des solutions compatibles en taille avec notre application, pour que leurs utilisations soient plus souples et de plus en plus autonomes, nous nous sommes attachés à résoudre plusieurs problèmes pour créer des sources ou alimentations compactes voir même intégrées. L'intégration des éléments passifs a toujours été le point le plus bloquant. Le développement des micro-technologies offre aujourd'hui de nouvelles perspectives pour la fabrication de condensateurs et micro-bobines sur silicium. Nous présentons dans cet article l'essentiel de l'état d'avancement des travaux menés dans ce domaine pour obtenir des éléments passifs de stockage performants.

\section{Problématique des microsystèmes.}

\subsection{Quelques généralités sur les microsystèmes.}

Le concept de microsystème ou MEMS (Micro Electro Mechanical Systems) est né à la fin des années 80 aux Etats-Unis, des actions conduites à l'Université de Berkeley pour intégrer sur une même puce de silicium, capteurs, traitement du signal et actionneurs. Il constitue une suite logique du progrès réalisé en microélectronique sur silicium, qui a permis la réduction des dimensions, l'augmentation de la qualité des composants et la réduction des coûts par l'emploi de procédés collectifs.

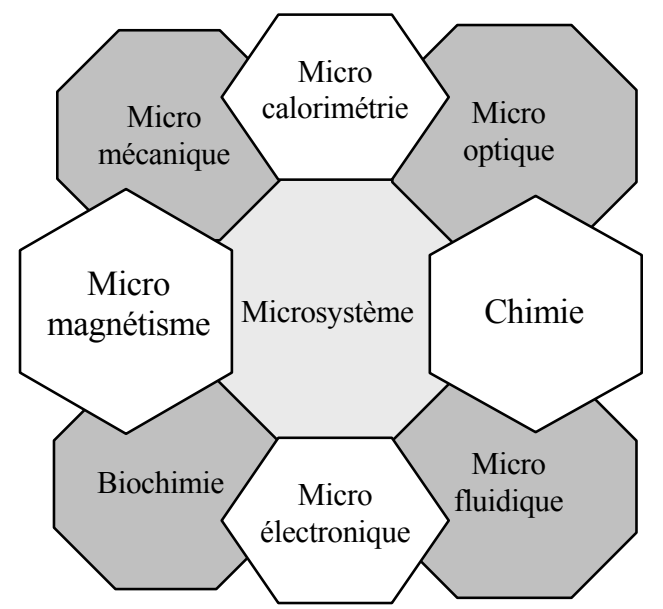

Figure 1. Multi-disciplinarité des microsystèmes

On peut ainsi, de manière compatible avec la présence des circuits intégrés, réaliser des structures 3D (membranes, 
cavités, poutres, etc...) et créer des micro-dispositifs dans le but d'effectuer une action ou capter l'environnement [4].

Depuis le début des années 90, de nombreux microsystèmes ayant des fonctionnalités très diverses ont été développés : gyroscopes, magnétomètres, microphones, micromoteurs, micro-pompes, etc... Bien qu'ayant nécessité un temps de développement plus important, à cause de leur plus grande complexité, ces microsystèmes ont eu beaucoup plus de difficulté à s'imposer sur le marché.

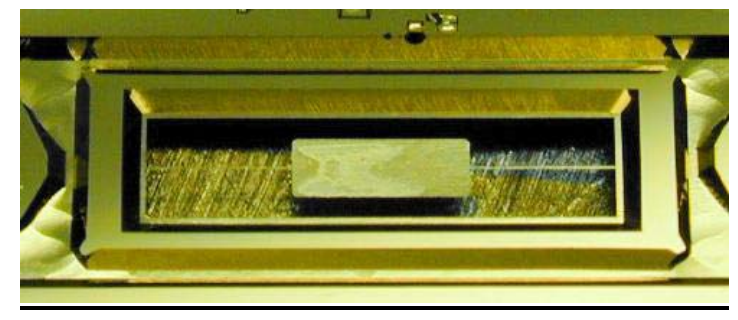

Figure 2. Micro-miroir à actionnement électrostatique [5].

D'autres microsystèmes ont, par contre, connu un grand succès commercial avec un marché important. Citons l'exemple des têtes d'imprimante à jet d'encre ainsi que les têtes de lecture/écriture pour disques durs et dans une moindre mesure, les systèmes de projection d'image [5-6].

\subsection{Actionnement des microsystèmes.}

Les microsystèmes comportent très souvent des pièces mobiles, qui, de part leur mise en mouvement, entraîne la réalisation d'une fonction. Par exemple, dans le cas du micro-miroir présenté en figure 2, l'application d'une tension sur une électrode entraîne la mise en rotation de la structure. Pour cela, différents types d'actionnement ont été développés, les principaux étant résumés dans le tableau 2 .

Les actionneurs électrostatiques sont couramment utilisés pour la mise en mouvement de membranes ou de poutres. Ils bénéficient de forces de rappel mécaniques qui viennent contrebalancer les forces électrostatiques qui font que les électrodes s'attirent et se rapprochent, quelle que soit la polarité des tensions appliquées aux électrodes.

\begin{tabular}{c|c|c|c|c} 
Mode & $\begin{array}{c}\text { Force } \\
\text { Pression }\end{array}$ & Fréqu. & $\begin{array}{c}\text { Tension } \\
\text { Courant }\end{array}$ & Puiss. \\
\hline Electrostatique & $P \alpha 2 \varepsilon \cdot \varepsilon_{0} \cdot\left(\frac{V}{e}\right)^{2}$ & $\begin{array}{c}0,1- \\
50 \mathrm{kHz}\end{array}$ & $\begin{array}{c}30- \\
1500 \mathrm{~V}\end{array}$ & $\mathrm{~mW}$ \\
\hline Magnétique & $F \alpha \mu_{0} \cdot B i$ & $\begin{array}{c}0,1- \\
50 \mathrm{kHz}\end{array}$ & $50 \mathrm{~mA}$ & $\mathrm{~W}$ \\
\hline Thermique & $P \alpha\left(\gamma_{1}-\gamma_{2}\right)$ & $\begin{array}{c}0,01- \\
1 \mathrm{kHz}\end{array}$ & $10 \mathrm{~V}$ & $0,1 \mathrm{~W}$ \\
\hline Piézo- & $P \alpha V$ & $\begin{array}{c}0,05- \\
30 \mathrm{kHz}\end{array}$ & $1 \mathrm{~V}$ & $\mathrm{~mW}$ \\
électrique & & &
\end{tabular}

Tableau 1. Comparaison des différents types d'actionnements.

Comme l'énergie stockée est directement proportionnelle à la capacité $C$ de l'actionneur, elle est indirectement proportionnelle à la distance entre les deux électrodes. Ce type d'actionnement sera donc destiné à des actionnements de pièces les plus rapprochées possibles.

L'actionnement magnétique, en opposition à l'actionnement électrostatique, peut générer des forces attractives et répulsives. Il présente l'avantage d'être sans fil et sans contact physique, en particulier dans le cas de la magnétostriction et présente la possibilité d'être intégré dans un packaging sous vide.

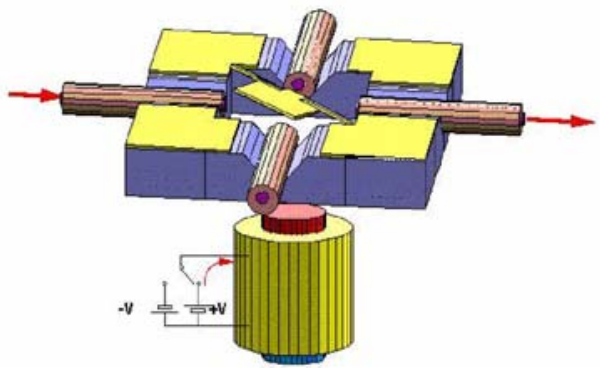

Figure 3. Micro-miroir à actionnement magnétique [7].

Mais l'inconvénient principal du mode magnétique est qu'il faut appliquer un champ magnétique externe par l'intermédiaire d'un aimant permanent, nuisant ainsi à la miniaturisation. De plus, dans le cas de la magnétostriction, des dépôts de couches magnétiques sont nécessaires. Ces solutions font appel à de nouveaux matériaux pour lesquels il faut développer des solutions d'élaboration de couches minces par des procédés compatibles avec les microtechnologies ainsi que des méthodes pour caractériser ces dernières.

L'actionnement thermique permet quant à lui une intégration monolithique du système, puisque des couches de silicium, polysilicium, oxyde de silicium et de métal suffisent pour constituer la partie mobile.

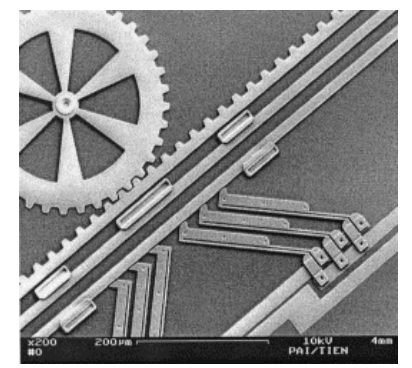

Figure 4. Détail d'un actionnement thermique [8].

Mais il présente des temps de réponse importants dus à l'importante inertie thermique et une forte consommation d'énergie. De plus, un vieillissement accéléré, du à l'échauffement, entraîne une dérive progressive des lois de commande dans le temps.

Enfin, l'actionnement piézoélectrique offre la possibilité d'atteindre des déplacements importants avec de faibles tensions d'actionnement et une grande résolution. [9]. Mais, le vieillissement et le phénomène de fatigue mécanique de telles couches ne sont pas encore bien maîtrisés.

\subsection{Problématique.}

Les batteries d'accumulateurs sont les éléments les plus fréquemment utilisés pour répondre à la nécessité d'alimentation en énergie des systèmes d'actionnement des microsystèmes. Mais ils ne peuvent délivrer les tensions requises par les différentes fonctions présentes dans ces systèmes. Une électronique de puissance d'interface est alors indispensable, dont la structure et les composants 
doivent être choisis en adéquation avec les particularités du contexte.

Les convertisseurs statiques basés sur des composants en commutation sont maintenant bien connus et universellement utilisés à des niveaux de puissance très variables. Dans le contexte qui nous préoccupe, ces convertisseurs sont très rarement utilisés dans des versions isolées (avec transformateur). En effet, aucun isolement de sécurité n'est requis car nous nous plaçons à très basse tension et le besoin d'une adaptation d'impédance dans de grandes proportions se fait rarement sentir.

\section{Structures de convertisseur adaptées.}

L'intégration des divers éléments composant un convertisseur statique est un des principaux enjeux aujourd'hui dans le domaine de l'électronique de puissance. Cependant, malgré des progrès constants dans ce domaine, il reste encore pas mal de verrous technologiques à résoudre pour obtenir des alimentations performantes n'occupant pas beaucoup de place.

\subsection{Généralités.}

Les points principalement résolus concernent essentiellement les semi-conducteurs qui permettent, par leurs commutations de contrôler le transfert de puissance [10]. Les composants actifs de puissance sont en effet de plus en plus performants pour convertir l'énergie avec de faibles pertes en conduction et en commutation. Ils sont de plus en plus faciles à commander avec des circuits de commande souvent simples, peu coûteux, totalement intégrables et ayant des consommations quasi-négligeables.

Evolution des volumes des convertisseurs de puissance et de la fréquence de commutation

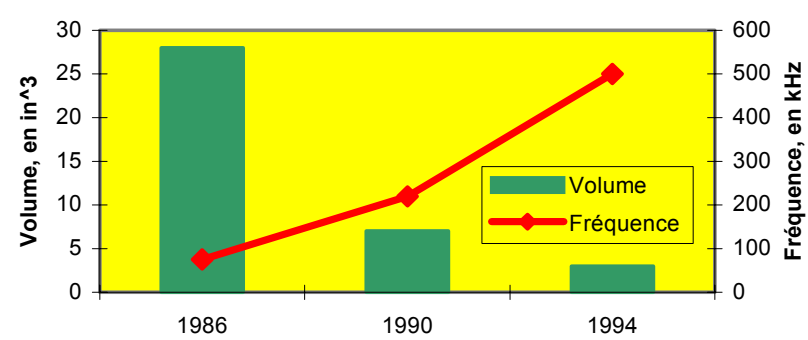

Figure 5. Evolution des volumes des convertisseurs de puissance et de leur fréquence de commutation [11].

De plus, des progrès importants dans l'augmentation des limites de leur fréquence de commutation ont été effectués. Ainsi, depuis une vingtaine d'années, elles ont augmenté graduellement de $75 \mathrm{kHz}$ au $\mathrm{MHz}$, ce qui a permis de réduire considérablement la totalité des tailles de tous les éléments du convertisseur statique [11].

\subsection{Structures adaptées.}

Les structures de base utilisées sont représentées dans la figure 6. Ainsi, la structure de la figure $6 \mathrm{a}$. est plutôt destinée à abaisser une tension, la structure de la figure 6 b. à l'élever. Les deux structures des figures $6 \mathrm{c}$. et $6 \mathrm{~d}$ sont plus polyvalentes, mais présentent des rendements plus faibles.
Nous retiendrons la figure 6a. comme structure de conversion possible pour assurer la fourniture d'une tension inférieure à $10 \mathrm{~V}$ et les structures $6 \mathrm{~b}$. et $6 \mathrm{~d}$. comme potentiellement intéressantes pour assurer la fourniture d'une tension élevée (de l'ordre de 100V).

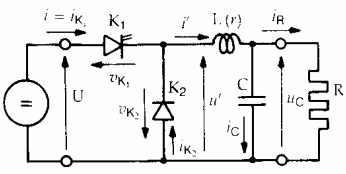

a.

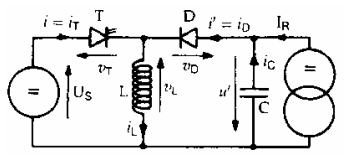

c.

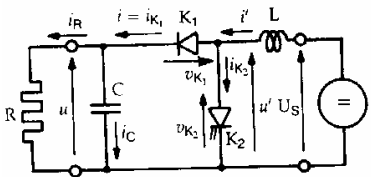

b.

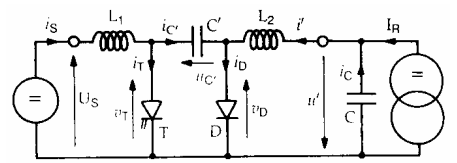

d.

Figure 6. Structure des convertisseurs à découpage nonisolés [12].

a. Hacheur abaisseur (Buck) - b. Hacheur élevateur (Boost) - c. Hacheur à stockage inductif abaisseurélevateur-inverseur (Buck-Boost) - d. Hacheur à stockage capacitif abaisseur-élevateur-inverseur (Cuck)

Selon la structure choisie, n'importe quel type de conversion peut être théoriquement réalisé.

\subsection{Les systèmes à découpages.}

Les systèmes à découpage exploitent les composants de puissance en commutation, ce qui élimine théoriquement l'inconvénient des systèmes linéaires qui avaient des rendements limités au maximum à $50 \%$. Ils doivent cependant inclure des filtres et des composants de stockage pour lisser les grandeurs de tension et de courant découpées et pour éliminer les effets harmoniques indésirables. Les différentes topologies présentées dans la figure 6 sont finalement très similaires, dans la mesure où elles utilisent le même couple «composant actif-diode» environné de différents composants passifs $L, C$. Globalement, le découpage reste relativement coûteux du fait de la présence de ces filtres.

Pour atteindre des rendements de convertisseur les plus élevés possibles, les composants actifs utilisés doivent présenter de faibles chutes de tension à l'état passant et de faibles capacités parasites, relativement à la puissance à convertir. Dans les faibles puissances, inférieures à 100W, le transistor MOSFET règne en maître. En effet, sa grille présente un faible niveau de seuil de déclenchement, ce qui permet de minimiser l'énergie perdue dans la commande. Par exemple, il tend à remplacer les diodes Schottky utilisées dans les redresseurs synchrones très faible tension, afin d'obtenir des tensions à l'état passant beaucoup plus basses, bien qu'il existe aujourd'hui des possibilités pour améliorer les performances de ces diodes [13]

Les autres composants sensibles présents dans ces montages sont les composants passifs utilisés pour stocker de l'énergie à l'échelle de la commutation. Leurs fréquences de travail font que leur valeur est généralement faible, de l'ordre de quelques micro-henrys. 


\section{Intégration des convertisseurs.}

Durant les deux dernières décennies, de grandes avancées ont marqué les domaines de la conception et de la fabrication des semi-conducteurs de puissance. En revanche, dans le même temps, l'intégration des composants réactifs n'a pas subi la même évolution, du fait de la difficulté d'obtenir des composants capables de supporter de fortes contraintes courant-tension, avec des valeurs d'inductances et de capacités telles qu'elles nécessitent la mise en œuvre de matériaux magnétiques et diélectriques. Ces problèmes peuvent être surmontés en s'orientant vers les faibles puissances, puisque les calibres en courant et en tension décroissent et que l'augmentation possible de la fréquence de découpage induit une réduction des valeurs des éléments inductifs et capacitifs.

\subsection{Cas des condensateurs.}

La valeur capacitive du condensateur est d'autant plus importante que la surface de l'armature et la constante diélectrique de l'isolant sont élevées et que l'épaisseur de l'isolant est faible. L'épaisseur de l'isolant est le principal facteur de limitation puisque sa réduction entraîne une diminution du champ électrique critique, une augmentation du courant de fuite et peut affecter la constante diélectrique.

La première voie consiste à augmenter la surface de l'armature. La technique de gravure profonde du silicium peut être exploitée dans la fabrication de condensateurs intégrés, en utilisant une topologie dite de tranchées, représentée en figure 7.

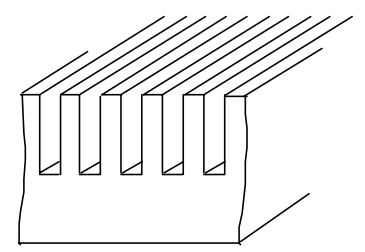

Figure 7. Topologie en tranchées d'un condensateur intégré [14].

Le rapport entre surface effective (réelle) et la surface apparente (de la puce) peut être relativement élevé et dépend de la profondeur de la gravure et de la distance inter-tranchées. Il en résulte un accroissement important de la capacité par unité de surface apparente.

Le tableau 2 donne quelques ordres de grandeurs de capacités sur la base d'une épaisseur d'isolant de 100A et d'un accroissement de surface d'un facteur 10, lié au principe des tranchées de $100 \mu \mathrm{m}$ de profondeur, $6 \mu \mathrm{m}$ de large avec une distance inter-tranchées d'environ $20 \mu \mathrm{m}$.

\begin{tabular}{c|c|c} 
Isolant & $\varepsilon_{\mathrm{r}}$ & $\mathrm{C}\left(\mu F / \mathrm{cm}^{2}\right)$ \\
\hline $\mathrm{SiO}_{2}$ & $\approx 3.9$ & 3.4 \\
\hline $\mathrm{Si}_{3} \mathrm{~N}_{4}$ & $\approx 7.5$ & 6.5
\end{tabular}

Tableau 2. Estimation des valeurs de capacités avec des diélectriques à base de silicium.

Il apparaît que l'utilisation de technologies de fabrication des topologies en «tranchées» permet de dégager des solutions susceptibles de répondre à nos besoins pour des applications de filtrage sous basse tension (quelques volts pour les micro-convertisseurs), avec des condensateurs à capacité constante de quelques microfarrad [14].

De nombreux travaux ont montré l'intérêt de l'emploi de condensateurs non linéaires en électronique de puissance, notamment dans la réalisation de circuits permettant d'améliorer les conditions de commutation des interrupteurs de puissance. En effet, ces condensateurs stockent moins d'énergie que leurs homologues à capacité constante et apportent donc un gain en termes de dissipation thermique et de durée de commutation.

Quelque soit la technologie de réalisation développée (accumulateurs électrochimiques, films minces à base d'oxynitrure, de céramique ou de polymère), la structure fondamentale d'un condensateur reste inchangée: une couche de diélectrique prise en sandwich entre deux couches conductrices. Dans cette optique, parmi les topologies intégrées permettant de maîtriser le non-linéarité de leur caractéristique $C(V)$ et procurant des valeurs de capacité et une tenue en tension compatibles avec l'application envisagée.

La deuxième voie consiste à remplacer les diélectriques classiques utilisés en micro-électronique $\left(\mathrm{SiO}_{2}, \mathrm{Si}_{3} \mathrm{~N}_{4}\right)$. Aujourd'hui, certains oxydes ferro-électriques (PZT, SBT, $B S T)$ peuvent présenter une constante diélectrique largement supérieure $(500<\varepsilon<1500)$ et permettent d'envisager des capacités surfaciques de plusieurs dizaines de $\mathrm{nF} / \mathrm{mm}^{2}$. Pour atteindre ces performances, des méthodes de dépôts associées doivent être développées.

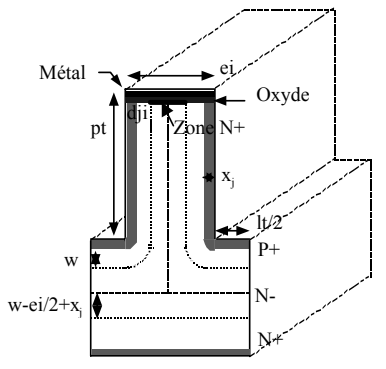

a.

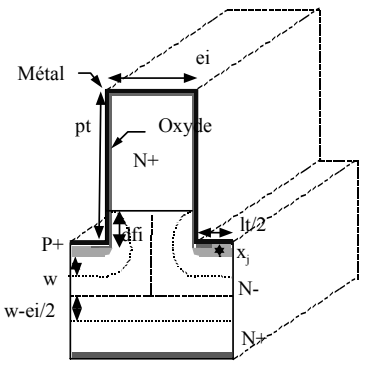

b.
Figure 8. Cellules capacitives élémentaires [14].

Ainsi, des associations $\mathrm{P}^{+} \mathrm{N} / \mathrm{MOS}$ permettent de réaliser des condensateurs non linéaires particulièrement bien adaptés à la réalisation de CALC à l'ouverture des interrupteurs de puissance.

Le principe de ces dispositifs est le contrôle de l'accès à une capacité de constante $C_{0}$ grâce à l'extension de zones de charge d'espace $(Z C E)$ de jonctions $\mathrm{P}^{+} \mathrm{N}$ convenablement disposées. L'évolution de ces $\mathrm{ZCE}$ due à l'application d'une tension inverse provoque, à partir d'une tension $V_{0}$, la mise en série d'une capacité de déplétion $C_{0}$. il y a donc une décroissance brutale de la valeur de la capacité à partir d'une valeur de tension définie par le dopage et la distance entre tranchées.

La réalisation des premiers dispositifs de test, basés sur des techniques de gravure profonde du silicium et sur le dépôt de silicium poly-cristallin dopé, ont permis de valider les simulations 2D et de vérifier la variation de capacité en fonction de la tension. 

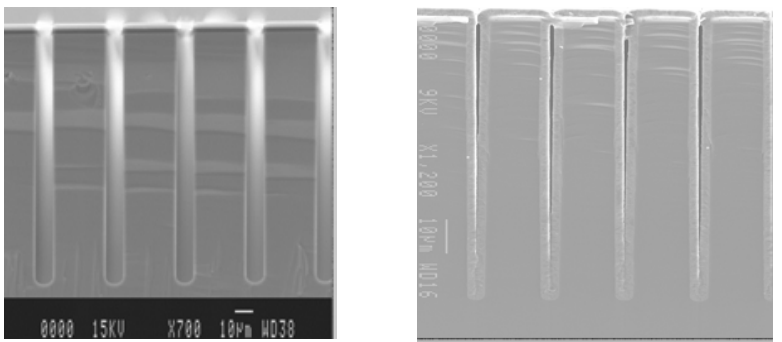

Figure 9. Vue en coupe des tranchées avec dépôt de polysilicium.

\subsection{Cas des inductances.}

L'intégration d'inductance sur silicium a d'abord été envisagée pour des applications autres que le stockage énergétique : micro-actionneur ou micro-capteur ou encore inductance de filtrage pour la RF. Mais, du fait des difficultés de réalisation technologique, de faibles valeurs d'inductances ont été atteintes jusqu'à ce jour, typiquement de l'ordre de la centaine de micro Henry. De ce fait, les diverses inductances réalisées jusque à présent ne sont pas conçues pour leur capacité de stockage d'énergie, mais pour leur fréquence de travail élevée. La plupart des structures fabriquées travaillent donc à très hautes fréquences, dans des circuits RF.

Depuis environ 5 ans, le besoin d'inductances intégrées pour réaliser des alimentations «on-chip» a relancé la recherche dans ce domaine. Les premières applications ont eu pour débouchés des inductances pour stockage de données sur disques magnétiques. En effet, les techniques actuelles de fabrication des microsystèmes (ie. Dépôt, gravure, etc...) permettent de réaliser, avec une grande précision de fabrication, les fils conducteurs ainsi que le noyau magnétique de la bobine, dans le cas où de faibles épaisseurs sont suffisantes.

\section{- Les inductances solénoïdes [15].}

Les inductances réalisées en discret et classiquement utilisées en stockage d'énergie à l'échelle d'une commutation possèdent en grande majorité une forme solénoïdale. Leur fabrication est ainsi facilitée puisque le fil conducteur peut être rapidement enroulé autour du tore magnétique. Mais cet empilement tri-dimensionnel est difficilement transposable aux faibles dimensions avec les techniques de micro-usinage et de dépôt offertes par les microtechnologies.

Les solutions proposées sur ce principe consistent soit en une solution hybride, où les fils conducteurs sont enroulés manuellement autour d'une couche magnétique, soit en une solution intégrée où les fils conducteurs sont constitués de cuivre déposé en plusieurs étapes sur un film en matériau magnétique.

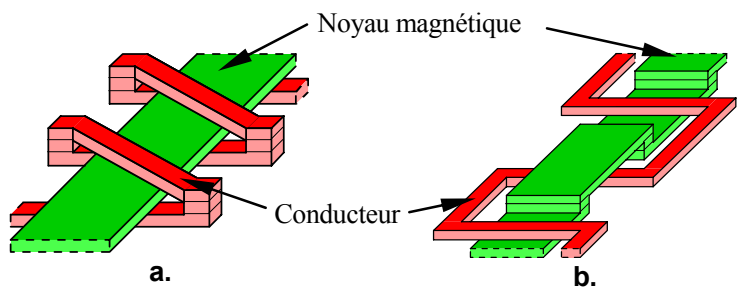

Figure 10. a. Bobine de type "toroïdal" - b. "serpentin".
L'avantage de ces solutions est qu'elles assurent un faible flux de fuite et donc une minimisation des interférences électromagnétiques.

- Les inductances à structure « mixte» [16].

Ces bobines sont basées sur un empilement de trois couches de matériaux. Mais, leur réalisation reste assez délicate d'un point de vue technologique, du fait de la nécessité de réaliser des plots entre les niveaux inférieur et supérieur. De ce fait, un nouveau type de structure dite mixte a été développée. Elle consiste en deux niveaux pour le conducteur et le noyau magnétique, limitant ainsi le nombre de masques nécessaires à sa fabrication. a.

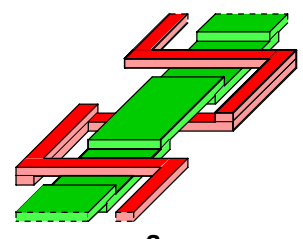

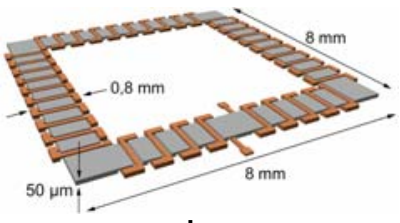

b.
Figure 11. Structure de type "mixte".

a. Principe - b. Géométrie de la bobine.

Si cette géométrie simplifie la réalisation, elle ne correspond pas à une solution optimale en terme d'encombrement. En effet, avec les dimensions du circuit magnétique reportées sur la figure $11 \mathrm{~b}$., le volume de matériau magnétique est approximativement de $1,2 \mathrm{~mm}^{3}$. de plus, ce volume sera encore augmenté par le feuilletage du circuit magnétique. Si l'on considère un foisonnement d'environ $50 \%$ entre le $\mathrm{NiFe}$ et la résine, le volume global du circuit magnétique feuilleté sera encore multiplié par 2.

D'autre part, la solution qui consiste à diminuer $\mu_{\mathrm{r}}$ est toute aussi pénalisante. En effet, si l'on veut conserver la même valeur d'inductance, il faudra d'augmenter le nombre de spires, et la résistance série du bobinage sera accrue.

Cette structure pourra toutefois trouver sa place dans des dispositifs ne nécessitant pas un stockage important d'énergie, comme c'est le cas dans les transformateurs.

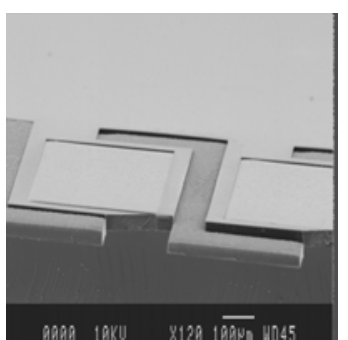

a.

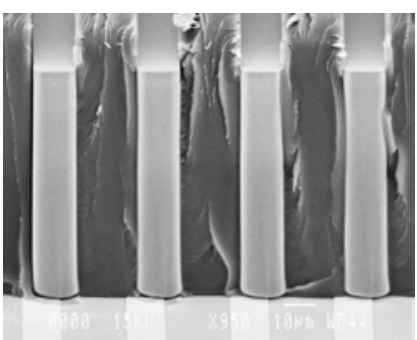

b.
Figure 12. Photographies MEB des premiers dépôts de matériaux réalisés au LAAS-CNRS [16].

- Les inductances spirales [17].

La plupart des inductances intégrées que l'on trouve dans la littérature possèdent une forme spirale. Elles sont réalisées soit sur un substrat isolant, soit magnétique ou bien entre deux couches de matériaux magnétiques.

Du fait des caractéristiques géométriques de la spirale, le flux magnétique possède deux composantes: une est parallèle à la surface du wafer et l'autre, perpendiculaire. Il est difficile d'intégrer à ce type de structure un tore magnétique pour guider le flux. En fait, ce dernier doit 
traverser la surface du substrat pour qu'il soit efficace. Sinon, il présente des fuites magnétiques très importantes. Une solution actuelle destinée à fabriquer un tore magnétique autour de la spire consiste à déposer successivement une couche de matériau magnétique, l'inductance puis une nouvelle couche de matériau magnétique, et enfin de refermer le tore. Cette méthode de fabrication de tores n'est pas optimisée pour des applications d'actionnement, car ils ne permettent pas un flux magnétique important.

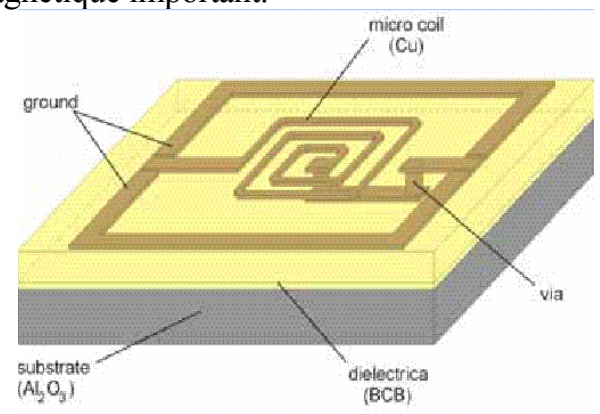

Figure 13. Exemples d'inductances spirales.

Pour des applications de stockage d'énergie, deux facteurs limitent l'amélioration des performances des inductances spirales. D'une part, le circuit magnétique doit être complété d'une couche métallique, afin de diminuer la réluctance magnétique et ainsi les lignes de champ parasites. D'autre part, la spire conductrice doit posséder une résistance la plus faible possible, pour réduire les pertes Joule $\mathrm{du}$ conducteur. Une des solutions utilisées actuellement consiste à encapsuler la bobine dans un tore magnétique réalisé en fer-nickel. Mais les techniques de dépôt de ce genre de matériau sont encore à améliorer, pour obtenir par exemple une bonne reproductibilité d'un process à l'autre.

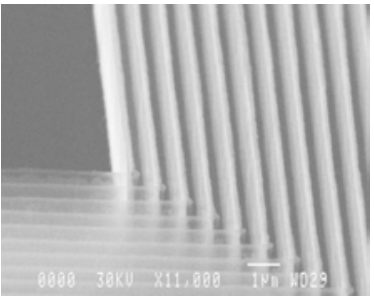

a.

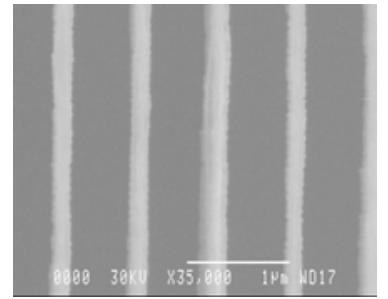

b.
Figure 13. Exemples d'inductances spirales réalisées à l'aide des nanotechnologies [16].

\section{Conclusion.}

La réalisation de convertisseurs statiques entièrement intégrés, permettant une entière compatibilité en taille avec des objets micro-usinés, passe par plusieurs étapes. D'une part, le choix de structures adéquates de conversion permettra à terme de réaliser des alimentations entièrement compatibles en taille et en consommation avec les applications. Pour cela, des travaux sur l'amélioration des matériaux et des filières technologiques de conversion de puissance doivent être renforcés pour s'adapter aux critères fort courant, très faible tension ou bien dans un autre domaine, forte tension, courant faible.
Il sera probablement nécessaire, pour arriver à une alimentation complètement intégrée, que les structures actuelles adaptées à des composants discrets ne puissent pas être directement miniaturisées. Il faudra ainsi faire preuve d'innovation dans des structures spécifiques réalisant la même fonction qu'en discret.

\section{Références}

[1] S. Raud, «Les Micro-sources d'énergie», Etats-Unis Microélectronique, $\mathrm{n}^{\mathrm{0}}$ 27, pp. 6-14, 2002.

[2] F. Forest, «Electronique de puissance basse tension, haut rendement pour les applications portables », Proceedings of Journées du Club EEA, Paris, pp. 97-106, 2002.

[3] B. Estibals, "Conception, réalisation et caractérisation de micro-miroirs optiques à déflexion localisée appliqués aux télécommunications optiques "), Thèse de l'INPT soutenue le 19 Décembre 2002.

[4] S. Tadigadapa, "Integration of micromachined devices and microelectronic circuits: techniques and challenges", Proceedings of the 43rd IEEE Midwest Symposium on Circuits and Systems, vol. 1, pp. 224-227, 2000.

[5] H. Camon, F. Larnaudie, "Fabrication, simulation and experiment of a rotating electrostatic silicon mirror with large angular deflection", Proceedings of the IEEE MEMS'2000, pp. 645-650, 2000.

[6] S. Renard, B. Estibals, V. Gaff, « Optical MEMS Components become Key Players in Optical Networks", Europhotonics, $n^{o}$ April/May01, pp. 33-35, 2001.

[7] G. Reyne, "Electromagnetic actuation for MOEMS : examples, advantages and drawbacks of MAGMAS", Journal of Magnetism and Materials, vol. 242-245, pp. 1119-1125, 2002.

[8] M. Hoffman, E. Vosges, "Bulk silicon micromachining for MEMS in optical communication systems", Journal of Micromechanic and Microengineering, vol. 12, pp. 349-360, 2002.

[9] T. Ono, Sensors and Actuators, vol. A21-A23, pp. 726-728, 1990.

[10]A-B. Frazier, R-O. Warrington, C. Friedrich, "the Miniaturization Technologies : Past, Present and Future", IEEE Transactions on Industrial Electronics, vol. 42, n ${ }^{\circ} 5$, pp. 423-430, 1995.

[11]A-W. Lofti, M-A. Wilkowski, "Issues and Advances in HighFrequency Magnetics for Switching Power Supplies", Proceedings of the IEEE, vol. 89, $\mathrm{n}^{\circ} 6, \mathrm{pp} .833-845,2001$.

[12]J-P. Ferrieux, F. Forest, «Alimentations à découpage, convertisseurs à résonance: principe, composants et

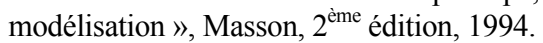

[13] T. Shimizu \& al. "100V Trench MOS Barrier Shottky Rectifier using Thick oxide layer (TO-TMBS)", Proceedings of International Symposium on Power semiconductor Devices, Osaka, 2001.

[14]H.Hakim ，J-P.Laur ，J-L.Sanchez , E.Scheid , P.Dubreuil, "Nonlinear capacitors integration", International Semiconductor Conference, Vol.1, pp.303-306, 2000.

[15] A. Lotfi, R-B. Van Dover, L. Schneemeyer, M. Steigerwald, "Micro-transformer devices using thin-film electroplated deposition", Proceedings of Power Electronics Specialists Conference 98, vol. 2 , pp 1511-1515, 1998.

[16]G. Troussier \& al., « Etude et réalisation d'éléments inductifs sur silicium dans la perspective d'intégration de microconvertisseurs », EPF'2002, pp.191-196, 2002.

[17] B. Estibals, C. Alonso, M-F. Shraif, H. Valderrama-Blavi, H. Camon, A. Martinez, "Design and integration of photovoltaic switching conversion chains", $6^{\text {th }}$ ESPC 2002, pp. 35-40, 2002. 